\title{
QUÍMICA ORGANOMETÁLICA DE SUPERFÍCIE APLICADA À PREPARAÇÃo DE CATALISADORES HETEROGÊNEOS BEM DEFINIDOS
}

Silvana I. Wolke e Regina Buffon*

Instituto de Química, Universidade Estadual de Campinas, CP 6154, 13083-970 Campinas - SP

Recebido em 21/9/01; aceito em 11/3/02

\begin{abstract}
SURFACE ORGANOMETALLIC CHEMISTRY APPLIED TO THE PREPARATION OF WELL DEFINED HETEROGENEOUS CATALYSTS. The study of the reactions of organometallic complexes with the surfaces of inorganic oxides, zeolites and metals constitutes the basis of Surface Organometallic Chemistry (SOMC). The basic rules of organometallic chemistry are often valid when applied to surfaces and well-defined surface organometallic complexes can be obtained. These complexes can be used as heterogeneous catalysts or, by controlled reactions, can be transformed in other species useful for a given catalytic reaction. In some cases, these catalysts exhibit higher activity and/or selectivity than their analogous molecular complexes.
\end{abstract}

Keywords: surface organometallic chemistry; heterogeneous catalysts; catalytic reactions.

\section{INTRODUÇÃO}

A química organometálica de superfície (Q.O.M.S.) é um campo da química que investiga a reatividade de complexos organometálicos e/ou de coordenação com superfícies de óxidos inorgânicos, zeólitas e metais ${ }^{1}$. Esta química está relacionada com a química de superfícies e com a ciência de materiais, e seu desenvolvimento é de grande relevância para a catálise.

O estudo sistemático da reatividade de complexos organometálicos em superfícies busca aplicar os conhecimentos da química de organometálicos e da catálise homogênea à catálise heterogênea, estudando a síntese de catalisadores sólidos a partir de análogos homogêneos ou de compostos organometálicos com um potencial para determinada reação catalítica. Nestes sistemas, conhecidos como sistemas heterogeneizados, o metal possui dois tipos de ligantes: $i$ ) ligantes provenientes do complexo de partida, que afetam a reatividade química, como ocorre na química em solução, e ii) ligantes rígidos, pertencentes à superfície, que conferem a natureza heterogênea ao complexo. Assim, são reunidas algumas das vantagens dos catalisadores heterogêneos e homogêneos, como a alta seletividade dos catalisadores em solução, a fácil separação dos catalisadores sólidos e, geralmente, a maior estabilidade térmica do catalisador ancorado em relação ao análogo solúvel. Adicionalmente, tem-se como vantagem a possível estabilização de intermediários pela superfície que, ao atuar como um ligante volumoso e rígido, proporciona um isolamento dos sítios e restringe a mobilidade das moléculas, dificultando reações de decomposição, principalmente de dimerização de espécies eletronica e coordenativamente insaturadas.

Os complexos organometálicos ancorados, obtidos via Q.O.M.S., podem ser utilizados diretamente em uma determinada reação catalítica ou podem ser transformados, mediante reações controladas, permitindo um controle do sítio ativo quanto à natureza, ao estado de oxidação, à natureza dos ligantes e ao número de coordenação adequado para uma determinada reação catalítica. Em alguns casos, demonstrou-se que o catalisador ancorado é mais ativo ou seletivo do que o análogo molecular ${ }^{2,3}$ ou do que o catalisador hete-

*e-mail: rbuffon@iqm.unicamp.br rogêneo que se pretende modelar ${ }^{4,5}$.

Enfim, os objetivos da Q.O.M.S. podem ser assim resumidos:

1. Compreensão, em nível molecular, das reações que ocorrem entre um complexo organometálico e os grupos presentes na superfície de um óxido inorgânico, de uma zeólita e de metais.

2. Caracterização dos complexos de superfície resultantes da reação de superfície, através de técnicas espectroscópicas e reatividade química.

3. Estudo da reatividade dos complexos de superfície a fim de verificar seu potencial para determinada reação catalítica.

4. Extensão dos conceitos da química de organometálicos à ciência de materiais.

\section{REGRAS BÁSICAS QUE GOVERNAM A REATIVIDADE DE COMPLEXOS ORGANOMETÁLICOS EM RELAÇÃO À SUPERFÍCIE DE ÓXIDOS INORGÂNICOS}

Embora a Q.O.M.S. seja um campo relativamente novo da química, já são conhecidas diversas reações que produzem complexos de superfície bem definidos ${ }^{1,6,7}$. As regras que governam a química molecular se aplicam às reações dos complexos organometálicos com os grupos reativos do óxido inorgânico, $\mathrm{M}_{\mathrm{x}} \mathrm{O}_{\mathrm{y}}$ (grupos hidroxilas da superfície, centros ácidos de Lewis, $\mathrm{M}^{\mathrm{n}+}$ e pontes $\left.\mathrm{M}-\mathrm{O}-\mathrm{M}\right)^{7}$. O controle da natureza e concentração das espécies reativas presentes na superfície pode ser conseguido pela variação da temperatura de prétratamento do óxido inorgânico ${ }^{8}$. Usualmente, os complexos de superfície resultantes são caracterizados por reatividade química e por técnicas espectroscópicas.

\section{REATIVIDADE DE CLUSTERS E DE COMPLEXOS METAL-CARBONILA EM BAIXO ESTADO DE OXIDAÇÃO}

Diferentes tipos de reações podem ocorrer entre clusters com ligantes carbonila ou complexos metal-carbonila com a superfície de óxidos inorgânicos, produzindo complexos de superfície bem definidos ${ }^{1,9}$. Em geral, o tipo de reação depende das características ácido-básicas do óxido inorgânico. 


\section{Interações ácido-base de Lewis}

Muito conhecida na química molecular, a formação de aduto ácido-base de Lewis é um dos modos mais simples de interação de complexos metal-carbonila com a superfície de óxidos inorgânicos. Em geral, o oxigênio básico do ligante carbonila (terminal ou em ponte) do complexo interage com um íon metálico, coordenativamente insaturado, da superfície. Também já foi observada a interação simultânea com os centros ácidos e básicos do suporte.

Por exemplo, os sítios ácidos e básicos de $\mathrm{Al}_{2} \mathrm{O}_{3}$ promovem a migração de metila nos complexos $\left[\mathrm{Mn}\left(\mathrm{CH}_{3}\right)(\mathrm{CO})_{5}\right]$ e $\left[\mathrm{Fe}(\mathrm{Cp})\left(\mathrm{CH}_{3}\right)(\mathrm{CO})_{2}\right]$, assim como fazem os ácidos de Lewis moleculares, como $\mathrm{AlBr}_{3}$, (Equação 1) ${ }^{10}$.



\section{Interação ácido-base de Brönsted}

Superfícies de óxidos básicos como alumina e magnésia são capazes de abstrair prótons coordenados a complexos metal-carbonila e clusters metálicos, produzindo complexos aniônicos de superfície. $\mathrm{O}$ óxido de magnésio, $\mathrm{MgO}$, que apresenta uma forte basicidade de Brönsted, desprotona o complexo $\left[\mathrm{H}_{2} \mathrm{O}(\mathrm{CO})_{4}\right]$, levando à formação de $\left[\mathrm{HOs}(\mathrm{CO})_{4}\right]^{-}$(Equação 2). Esse ânion é adsorvido na superfície por uma interação ácido-base de Lewis do oxigênio da carbonila com íons $\mathrm{Mg}^{2+}$ da superfície da $\mathrm{MgO}$, numa interação análoga à apresentada pelo par iônico $\mathrm{Na}\left[\mathrm{HOs}(\mathrm{CO})_{4}\right]$ em $\mathrm{THF}^{11}$.

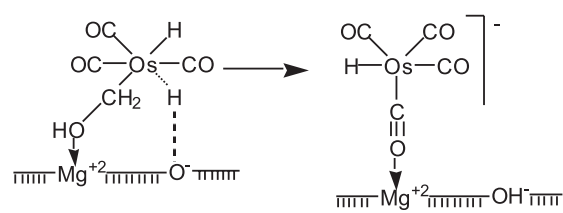

\section{Ataque nucleofílico a CO coordenado}

Carbonilas metálicas do grupo 8 , tais como $\left[\mathrm{M}_{3}(\mathrm{CO})_{12}\right],(\mathrm{M}=$ $\mathrm{Fe}, \mathrm{Ru}, \mathrm{Os}$ ) sofrem ataque nucleofílico pelos grupos hidroxilas da superfície de óxidos básicos (magnésia parcialmente hidroxilada, alumina e óxido de zinco ou de lantânio) ${ }^{12}$. A reação ocorre com a formação dos respectivos hidretos aniônicos e a eliminação de $\mathrm{CO}_{2}$, de maneira análoga à observada em solução (Equação 3).

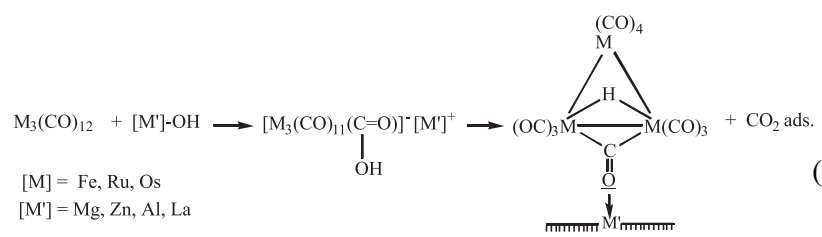

\section{Adição Oxidativa}

Clusters metálicos como $\left[\mathrm{Ru}_{3}(\mathrm{CO})_{12}\right]$ ou $\left[\mathrm{Os}_{3}(\mathrm{CO})_{12}\right]$ reagem com óxidos inorgânicos que possuam grupos $\mathrm{OH}$ levemente ácidos, como silanóis, via adição oxidativa, com a formação de um hidreto em ponte (Equação 4) ${ }^{13}$.
O complexo de superfície $\left[\mathrm{Os}_{3}(\mathrm{CO})_{10}(\mu-\mathrm{H})(\mu-\mathrm{OSi} \equiv)\right]$, resultante da reação do cluster $\left[\mathrm{Os}_{3}(\mathrm{CO})_{12}\right]$ com a superfície de $\mathrm{SiO}_{2}$, foi caracterizado por uma variedade de técnicas, o que permitiu a completa elucidação de sua estrutura ${ }^{14}$. A mesma reação ocorre em $\mathrm{Al}_{2} \mathrm{O}_{3}{ }^{13}$.

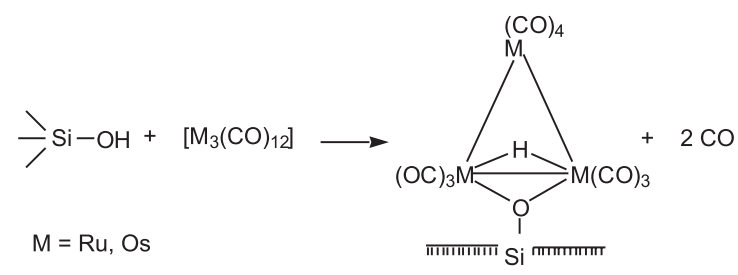

\section{REATIVIDADE DE COMPLEXOS ORGANOMETÁLICOS EM ALTO ESTADO DE OXIDAÇÃO}

Nesta seção será apresentada a reatividade de complexos organometálicos, do tipo metal-alquila, metal-alquilideno e metalalquilidino em relação à superfície de óxidos inorgânicos.

\section{Clivagem eletrofílica de ligações metal-carbono}

Este tipo de reação ocorre quando um complexo metal-alila ou metal-alquila reage com grupos $\mathrm{OH}$ eletrofílicos da superfície de um óxido inorgânico, liberando o correspondente alcano ou olefina.

\section{Complexos metal-alila}

A reatividade de complexos metal-alila de vários elementos ( $\mathrm{Zr}$, $\mathrm{Hf}, \mathrm{Nb}, \mathrm{Cr}, \mathrm{Mo}, \mathrm{W}, \mathrm{Rh}, \mathrm{Ni}, \mathrm{Pd}, \mathrm{Pt}$ ) foi estudada com óxidos inorgânicos, principalmente com sílica ${ }^{3,15}$. O complexo $\left[\mathrm{Rh}\left(\eta^{3}-\right.\right.$ $\left.\mathrm{C}_{3} \mathrm{H}_{5}\right)_{3}$ ] reage com sílica formando um complexo de superfície bem definido, (Equação 5) ${ }^{16}$.

$\equiv \mathrm{SiOH}+\left[\mathrm{Rh}\left(\eta^{3}-\mathrm{C}_{3} \mathrm{H}_{5}\right)_{3}\right] \rightarrow\left[\equiv \mathrm{SiO}-\mathrm{Rh}\left(\eta^{3}-\mathrm{C}_{3} \mathrm{H}_{5}\right)_{2}\right]+\mathrm{C}_{3} \mathrm{H}_{6}$

A reação ocorre à temperatura ambiente e segue sempre a mesma estequiometria, independentemente da temperatura de pré-tratamento da sílica utilizada. O monitoramento por IV in situ e a liberação de propeno da reação de superfície mostram que são os grupos $\mathrm{OH}$ da sílica que atacam as ligações Rh-C, clivando-as ${ }^{16}$. Adicionalmente, por IV, foi verificado que os ligantes alila permanecem em coordenação $\eta^{3}$ ao $\mathrm{Rh}{ }^{16}$.

Baseados nos resultados de modelagem molecular, usando comprimentos e ângulos de ligação idênticos aos do complexo modelo $\left[\mathrm{Rh}(\mu-\mathrm{OH})\left(\eta^{3}-\mathrm{C}_{3} \mathrm{H}_{5}\right)_{2}\right]_{2}$, e nos dados obtidos por IV-TF foram propostas estruturas para $\left[\equiv \mathrm{SiO}-\mathrm{Rh}\left(\eta^{3}-\mathrm{C}_{3} \mathrm{H}_{5}\right)_{2}\right]$ em que o átomo de $\mathrm{Rh}$ de superfície está coordenado a um átomo de oxigênio, aceitando a doação eletrônica de um silanol ou siloxano adjacente a fim de alcançar a configuração de 18 e- (Figura 1$)^{16}$.

Técnicas como espectroscopia Raman, espectroscopia de RMN no estado sólido dos núcleos ${ }^{1} \mathrm{H}\left[{ }^{1} \mathrm{H}-\mathrm{RMN}-\mathrm{CRAMPS}\right.$ ("Combined
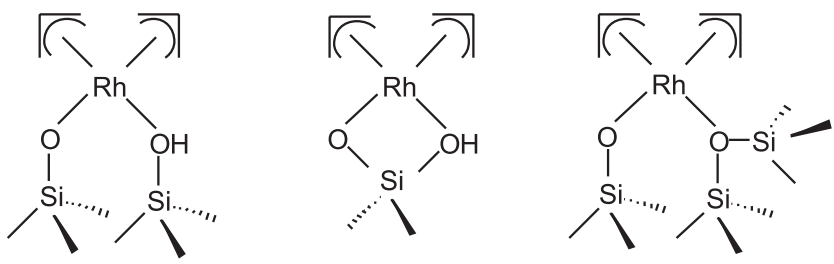

Figura 1. Estruturas do complexo $\left[\mathrm{Rh}\left(\eta^{3}-\mathrm{C}_{3} \mathrm{H}_{5}\right)_{3}\right]$ suportado em sílica ${ }^{16}$ 
rotation and multiple pulse sequence")] e ${ }^{13} \mathrm{C}\left({ }^{13} \mathrm{C}-\mathrm{RMN}-\mathrm{CP}-\mathrm{MAS}\right)$ de uma amostra enriquecida em ${ }^{13} \mathrm{C},\left[(\equiv \mathrm{SiO}) \mathrm{Rh}\left(\mathrm{CH}_{2} \mathrm{CH}^{13} \mathrm{CH}_{2}\right)\right]$, e XPS foram usadas para complementar a caracterização das espécies de superfície ${ }^{16}$.

Diferentemente do complexo $\left[\mathrm{Rh}\left(\eta^{3}-\mathrm{C}_{3} \mathrm{H}_{5}\right)_{3}\right]$, foi verificado que a temperatura de pré-tratamento da sílica (relacionada com a concentração de grupos silanóis) influencia a reatividade do complexo $\left[\mathrm{Mo}\left(\eta^{3}\right.\right.$ $\left.\mathrm{C}_{3} \mathrm{H}_{5}\right)_{4}$ ]. Em sílicas tratadas entre 300 e $400{ }^{\circ} \mathrm{C}$, a reação ocorre com os grupos silanóis, e com sílicas tratadas acima dos $500{ }^{\circ} \mathrm{C}$, o complexo também reage com os grupos siloxanos (Equações 6 e 7) ${ }^{15}$.



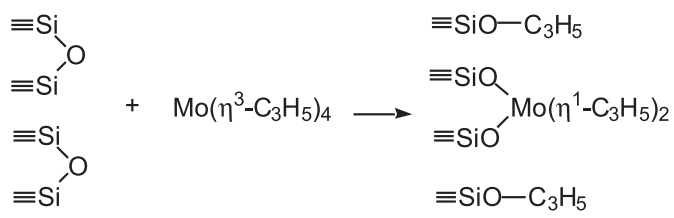

No espectro no IV-TF dos complexos de superfície foi observada uma banda em $1650 \mathrm{~cm}^{-1}$, atribuída ao $\delta_{(\mathrm{C}=\mathrm{C})}$ do ligante alila coordenado ao Mo por uma ligação $\sigma$. Isto sugere a mudança de coordenação $\left(\eta^{3} \rightarrow \eta^{1}\right)$ dos ligantes alila após a reação do complexo com os grupos $\mathrm{OH}$ da sílica ${ }^{15}$. Entretanto, estudos por espectroscopia fotoacústica no infravermelho, RMN no estado sólido $\left({ }^{1} \mathrm{H} \mathrm{e}{ }^{13} \mathrm{C}\right)$, e cálculos teóricos ${ }^{17}$ sugerem a presença de ligantes alila coordenados $\eta^{3}$ e $\eta^{1}$ ao Mo. Através de espectroscopia eletrônica ${ }^{15}$ e XPS ${ }^{18}$, foi mostrado que o molibdênio mantém o estado de oxidação (IV) na espécie de superfície.

\section{Complexos metal-alquila}

$\mathrm{Na}$ classe dos complexos metal-alquila, a reação do complexo zircônio-tetraneopentila com os grupos $\mathrm{OH}$ de uma sílica desidroxilada a $500{ }^{\circ} \mathrm{C}, \mathrm{SiO}_{2}$ [500], ilustra a clivagem de ligações $\mathrm{M}-\mathrm{C}$ por grupos $\mathrm{OH}$ da sílica (Equação 8$)^{19}$.
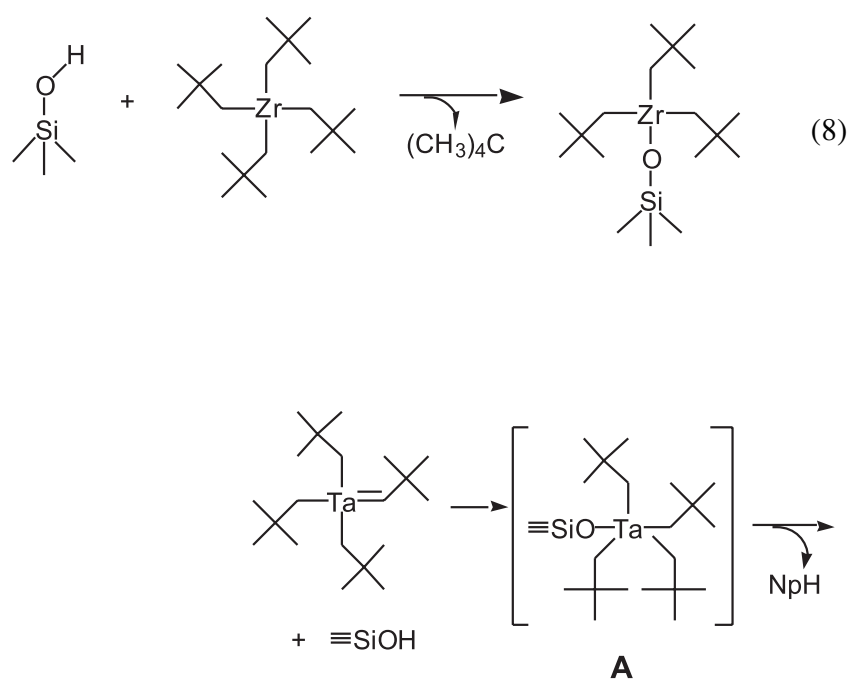

A estrutura do complexo de superfície foi proposta com base em resultados de análise quantitativa dos gases liberados durante a reação de ancoramento, espectroscopia no IV-TF in situ, análise elementar $^{19}$ e análise por EXAFS ${ }^{20}$.

Recentemente, foram sintetizados metalocenos relativamente bem definidos de zircônio, a partir de $\left[\mathrm{Zr}\left(\mathrm{Cp}^{*}\right)\left(\mathrm{CH}_{3}\right)_{3}\right], \mathrm{Cp}^{*}=$ pentametilciclopentadienila, e $\left[\mathrm{Zr}(\mathrm{Cp})_{2}\left(\mathrm{CH}_{3}\right)_{2}\right]$, em suportes como $\mathrm{SiO}_{2}, \mathrm{SiO}_{2}-\mathrm{Al}_{2} \mathrm{O}_{3}, \mathrm{Al}_{2} \mathrm{O}_{3}$ e $\mathrm{Nb}_{2} \mathrm{O}_{5}{ }^{21}$. Os grupos $\mathrm{OH}$ atacam uma ligação $\mathrm{Zr}-\mathrm{CH}_{3}$, clivando-a e liberando metano, gerando as espécies [M$\left.\mathrm{O}-\mathrm{Zr}\left(\mathrm{Cp}^{*}\right)\left(\mathrm{CH}_{3}\right)_{2}\right]$ e $\left[\mathrm{M}-\mathrm{O}-\mathrm{Zr}(\mathrm{Cp})_{2}\left(\mathrm{CH}_{3}\right)\right], \mathrm{M}=\mathrm{Si}, \mathrm{Nb}$, respectivamente. $\mathrm{Em} \mathrm{Al}_{2} \mathrm{O}_{3}[500]$ e em $\mathrm{SiO}_{2}-\mathrm{Al}_{2} \mathrm{O}_{3}[500]$ também foi observada a formação de complexos em que uma metila do complexo de superfície interage com um centro ácido de Lewis $(\mathrm{Al})$ dos suportes, por exemplo, $\left\{[\mathrm{Al}]_{2}-\mathrm{OZr}\left(\mathrm{Cp}^{*}\right)\left(\mathrm{CH}_{3}\right)\left(\mu \mathrm{CH}_{3}\right)-[\mathrm{Al}]\right\}^{21}$.

\section{Protonação de ligações $M=C, M \equiv C, M \equiv N$ e $M=N$.}

\section{Protonação de ligações $M=C$ de complexos metal-alquilideno}

$\mathrm{O}$ complexo $\left[\mathrm{Ta}\left(=\mathrm{CH}^{\mathrm{t}} \mathrm{Bu}\right)\left(\mathrm{CH}_{2}{ }^{\mathrm{t}} \mathrm{Bu}\right)_{3}\right]$ ao reagir com sílica produz complexos Ta-alquilideno de superfície, $\left[(\equiv \mathrm{SiO}) \mathrm{Ta}\left(=\mathrm{CH}{ }^{\mathrm{t}} \mathrm{Bu}\right)\left(\mathrm{CH}_{2}{ }^{\mathrm{t}} \mathrm{Bu}\right)_{2}\right], \mathbf{A}, \mathrm{e}\left[(\equiv \mathrm{SiO})_{2} \mathrm{Ta}\left(=\mathrm{CH} \mathrm{Hu}^{\mathrm{B}}\right)\left(\mathrm{CH}_{2}{ }^{\mathrm{t}} \mathrm{Bu}\right)\right]$, B. A formação de $\mathbf{A}$ ou $\mathbf{B}$ pode ser controlada de acordo com a temperatura de pré-tratamento da sílica ${ }^{8}$. O pré-tratamento a $700{ }^{\circ} \mathrm{C}$ leva à formação da espécie $\mathbf{A},(>90 \%)$, a $300^{\circ} \mathrm{C}$ é formada a espécie $\mathbf{B}$, (>90\%), e a $500{ }^{\circ} \mathrm{C}$ é formada uma mistura de espécies $\mathbf{A},(65 \pm$ $10 \%)$ e $\mathbf{B},(35 \pm 10 \%)^{8}$. Estudos de espectroscopia no IV in situ e a liberação de neopentano da reação indicaram que o complexo reage com os grupos $\mathrm{OH}$ da $\mathrm{SiO}_{2}{ }^{22}$. A presença do ligante neopentilideno coordenado ao átomo de tântalo foi mostrada por reação do tipo Wittig com acetona, típica para a detecção e quantificação de fragmentos alquilidenos ${ }^{23}$, em que foi detectada a olefina correspondente, 2,4,4,trimetil-2-penteno (Equação 9).

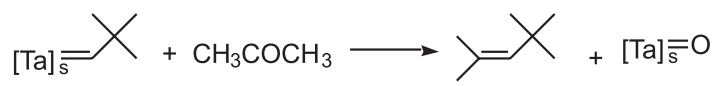

Um estudo detalhado de reatividade sugere que não acontece uma simples clivagem de ligações Ta-C por grupos $\mathrm{OH}$. Primeiramente, ocorreria a adição de um silanol à ligação $\mathrm{Ta}=\mathrm{C}$, formando um intermediário $\left[(\equiv \mathrm{SiO}) \mathrm{Ta}\left(\mathrm{CH}_{2}{ }^{ } \mathrm{Bu}\right)_{4}\right]$ que, por abstração de hidrogênio $\alpha$, produziria o complexo alquilideno de superfície A. Parte deste complexo sofreria a adição de um segundo grupo silanol, gerando o complexo de superfície B (Esquema 1) ${ }^{22}$.

\section{Complexos metal-alquilidinos}

Em solução, complexos tungstênio-alquilidinos podem ser protonados por ácidos de Brönsted formando complexos tungstênio- 
alquilideno ${ }^{24}$. O mesmo tipo de reação acontece em superfície, produzindo alquilidenos de superfície relativamente bem definidos via adição de um grupo silanol de sílica à ligação metal-alquilidino dos complexos de tungstênio $\left[\mathrm{W}\left(\equiv \mathrm{C}^{t} \mathrm{Bu}\right)\left(\mathrm{O}^{\mathrm{t}} \mathrm{Bu}\right)_{3}\right]$, (1) $\left[\mathrm{W}\left(\equiv \mathrm{C}^{\mathrm{t}} \mathrm{Bu}\right)\right.$ $\left.\left(\mathrm{CH}_{2}{ }^{\mathrm{t}} \mathrm{Bu}\right)_{3}\right],(2)$ e $\left[\mathrm{W}\left(\equiv \mathrm{C}{ }^{\mathrm{t} B u}\right)(\mathrm{Cl})_{3}(\mathrm{dme})\right],(3)$, dme =dimetoxietano, (Equação 10) ${ }^{25}$.

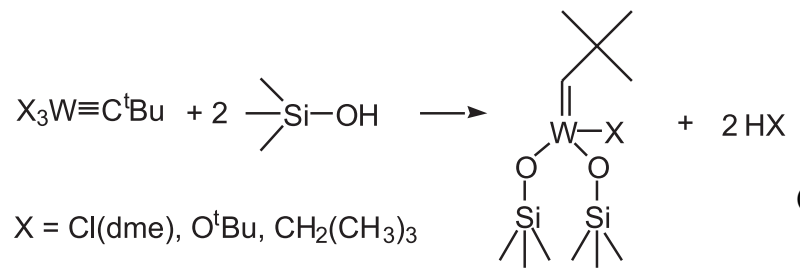

Nesse caso, usando como suporte $\mathrm{SiO}_{2}$, que apresenta somente sítios ácidos de Brönsted, foram formadas espécies relativamente bem definidas. Entretanto, a reatividade dos complexos (2) e (3) com os grupos $\mathrm{OH}$ de $\mathrm{Nb}_{2} \mathrm{O}_{5}$, que apresenta sítios ácidos de Brönsted e de Lewis, é mais complexa ${ }^{26}$. Além das espécies resultantes da reação de protonação da ligação $\mathrm{W} \equiv \mathrm{C}$, foi sugerida a formação de espécies provenientes de reações de protonação da ligação $\mathrm{W}=\mathrm{C}$ e de ataque eletrofílico dos prótons da superfície aos ligantes cloreto e neopentila (Esquema 2).
(A)

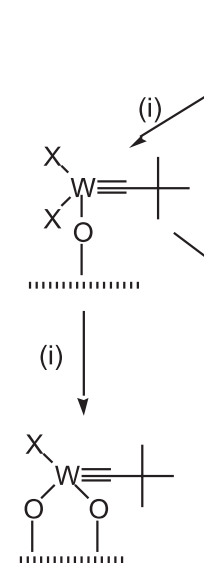

$\left(A^{\prime}\right)$



(B)

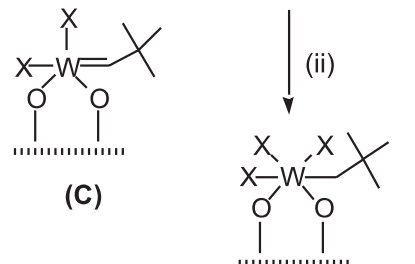

(B')

$$
\mathrm{X}=\mathrm{Cl}(\mathrm{dme}), \mathrm{CH}_{2}\left(\mathrm{CH}_{3}\right)_{3}
$$

Esquema 2. Reatividade do complexo $\left[\mathrm{W}\left(\equiv \mathrm{C}^{t} \mathrm{Bu}\right)(\mathrm{X})_{3}\right]$ em $\mathrm{Nb}_{2} \mathrm{O}_{5}^{26}$

As espécies A, A', B, B' e C foram propostas a partir de resultados de reatividade química (Esquema 2$)^{26}$.

\section{Complexo metal-nitreno}

O complexo $\left[\mathrm{Mo}(\equiv \mathrm{N})(\mathrm{Np})_{3}\right]$, ao reagir com a sílica, sofre adição 1,2 de silanóis da superfície à ligação $\mathrm{M} \equiv \mathrm{N}(\text { Equação } 11)^{27}$.

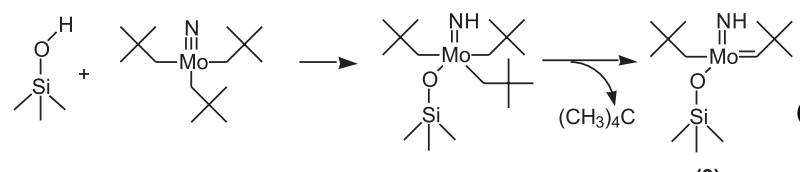

(2)

(3)

A reação foi proposta com base nos espectros no IV in situ. O complexo de superfície apresenta bandas entre 3000 e $2850 \mathrm{~cm}^{-1} \mathrm{e}$ entre 1500 e $1300 \mathrm{~cm}^{-1}$, atribuídas ao $\mathrm{v}_{(\mathrm{CH})}$ e ao $\delta_{(\mathrm{CH})}$ dos ligantes neopentila, e em $3370 \mathrm{~cm}^{-1}$, atribuída ao $v_{(\mathrm{NH})}$. Quando a reação foi efetuada com sílica deuterada, a banda em $3370 \mathrm{~cm}^{-1}$ foi deslocada para $2465 \mathrm{~cm}^{-1}$, frequiência esperada para o intercâmbio isotópico $\mathrm{H} / \mathrm{D}$, comprovando a adição 1,2 dos silanóis à ligação $\mathrm{M} \equiv \mathrm{N}$. Posteriormente, o complexo (3) é gerado por abstração de hidrogênio na posição $\alpha$ com liberação de neopentano (Equação 11). A presença do ligante neopentilideno foi verificada por reatividade com acetona quando foi produzido 2,4,4-trimetil-2-penteno quantitativamente ${ }^{27}$.

\section{Complexos metal-imido}

Os complexos metal-imido $\left[\mathrm{V}\left(=\mathrm{N}^{t} \mathrm{Bu}\right)(\mathrm{Np})_{3}\right]$ e $\left[\mathrm{V}\left(=\mathrm{N}^{\mathrm{t} B u}\right)\left(\mathrm{O}^{\mathrm{t} B u}\right)\right.$ $(\mathrm{Np})_{2}$ ] sofrem adição 1,2 de grupos $\mathrm{OH}$ de sílica à ligação $\mathrm{V}=\mathrm{N}$ (Esquema 3, espécie $\mathbf{C})^{28,29}$. A reação, proposta com base nos espectros de IV-TF in situ, ocorre em menor extensão, mas este é o primeiro relato de sua ocorrência em superfícies. Majoritariamente, ocorre ataque eletrofílico de grupos $\mathrm{OH}$ à ligação V-C (Esquema 3, espécies A e B).

\section{Interação ácido-base de Lewis}

O complexo $\left[\mathrm{WO}(\mathrm{ONp}) \mathrm{Np}_{3}\right]$ reage com o óxido de nióbio, desidroxilado a $300{ }^{\circ} \mathrm{C}$, formando, predominantemente, um aduto ácido-base de Lewis, espécie B (Esquema 4) ${ }^{30}$.

Outras espécies foram formadas sobre a superfície: $i$ ) espécie $\mathbf{A}$, produto do ataque de grupos $\mathrm{OH}$ à ligação $\mathrm{M}-\mathrm{C}$ do complexo de partida; ii) o neopentilideno de superfície $\mathbf{C}$, resultante da abstração de hidrogênio $\alpha$, fotoiniciada e induzida pela presença de ácidos de



(A)



(B)

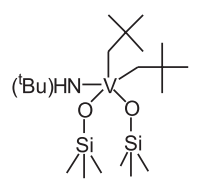

(C)
Esquema 3. Reatividade do complexo $\left[V\left(=N^{t} \mathrm{Bu}\right)(\mathrm{Np})_{3}\right]$ em $\mathrm{SiO}_{2}$ pré-tratada a $500{ }^{\circ} \mathrm{C}^{29}$

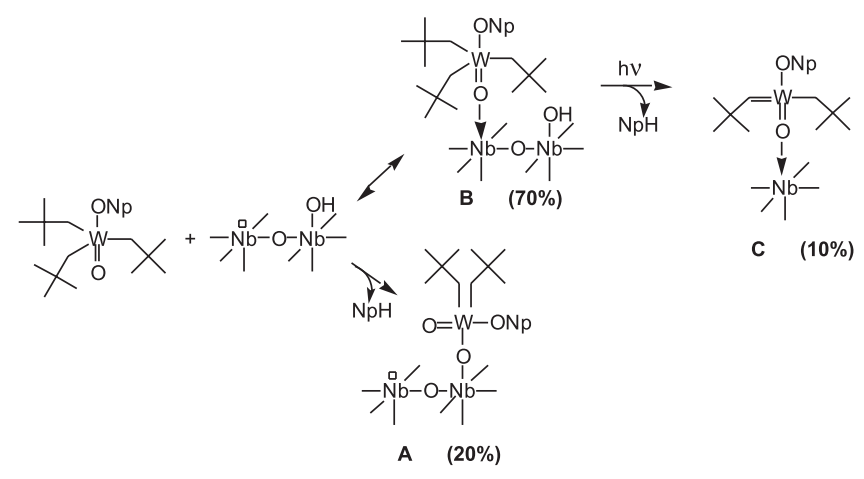

Esquema 4. Reatividade de [WO(ONp) $\left.\mathrm{Np}_{3}\right]$ em $\mathrm{Nb}_{2} \mathrm{O}_{5}{ }^{30}$ 
Lewis, como ocorre em solução ${ }^{31}$. A formação de $\mathbf{C}$ foi confirmada por reação do tipo Wittig com acetona quando foi detectada a olefina correspondente, 2,4,4-trimetil-2-penteno ${ }^{30}$.

Os complexos $\left[\mathrm{Mo}\left(=\mathrm{CHCPhMe}_{2}\right)(=\mathrm{NAr})(\mathrm{OR})_{2}\right] ; \mathrm{OR}=$ $\left(\mathrm{OC}(\mathrm{Me})_{3}\right)$ e $\left(\mathrm{OC}(\mathrm{Me})\left(\mathrm{CF}_{3}\right)_{2}\right), \mathrm{Ar}=2,5$-diisopropilfenila, formam adutos ácido-base de Lewis com um ou dois grupos silanóis de sílica desidroxilada a $500{ }^{\circ} \mathrm{C}$ (Figura 2), de acordo com estudos de espectroscopia no IV in situ e reatividade química ${ }^{32}$. Neste caso, o par de elétrons do oxigênio do grupo silanol estabilizaria os complexos, como acontece em solução com o complexo $\left[\mathrm{Mo}\left(=\mathrm{CHCPhMe} \mathrm{C}_{2}\right)\right.$ $\left.(=\mathrm{NAr})\left(\mathrm{OC}(\mathrm{Me})\left(\mathrm{CF}_{3}\right)_{2}\right)_{2}\right]$ em presença de solventes coordenantes como o THF e dimetoxietano ${ }^{33}$.

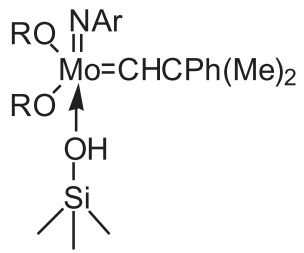

(A)

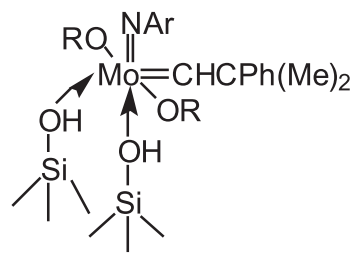

(A')

$$
\begin{aligned}
& \mathrm{OR}=\mathrm{O}^{\mathrm{t}} \mathrm{Bu} ; \mathrm{CMe}\left(\mathrm{CF}_{3}\right)_{2} \\
& \mathrm{Ar}=2,5 \text {-disopropilfenila }
\end{aligned}
$$

Figura 2. Adutos ácido-base de Lewis dos complexos $\left[\mathrm{Mo}\left(=\mathrm{CHCPhMe}_{2}\right)(=\mathrm{NAr})(\mathrm{OR})_{2}\right]$ em sílica ${ }^{32}$

\section{REATIVIDADE DE COMPLEXOS ORGANOMETÁLICOS DE SUPERFÍCIE}

A reatividade de complexos organometálicos de superfície também pode ser interpretada considerando as reações da química molecular ${ }^{34}$. Em princípio, essas reações podem ser consideradas como etapas elementares em catálise heterogênea. Muitos trabalhos foram publicados nesta área e algumas revisões apresentam reações estequiométricas e reações catalíticas ${ }^{7,35}$.

\section{Reatividade com hidrogênio}

Hidretos metálicos são intermediários-chave em várias reações catalíticas. Através da Q.O.M.S., sintetizaram-se hidretos metálicos de superfície ( $\mathrm{Zr}$, Ta, Ti, Hf) bem definidos, extremamente deficientes de elétrons, estabilizados pela superfície de óxidos inorgânicos, ativos em reações catalíticas em que seus análogos moleculares não apresentam atividade ${ }^{4,36}$

Os complexos M-hidreto foram sintetizados através das reações dos complexos de superfície com hidrogênio molecular. A caracterização foi efetuada por IV in situ (Tabela 1), reatividade química (Es- quema 5) e quantificação dos produtos liberados das reações de superfície $^{37,38}$. Adicionalmente, os hidretos de zircônio e de tântalo foram caracterizados por $\mathrm{EXAFS}^{20,39}$.

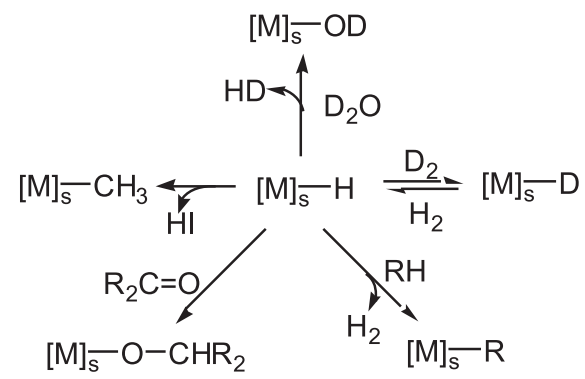

$\mathrm{M}=\mathrm{Zr}, \mathrm{Ta}, \mathrm{Ti}$

Esquema 5. Reatividade de complexos M-hidreto de superficie

A presença do ligante tbutilimido no complexo de superfície $\left[(\equiv \mathrm{SiO})_{2} \mathrm{~V}\left(=\mathrm{N}^{t} \mathrm{Bu}\right)\left(\mathrm{CH}_{2} \mathrm{C}\left(\mathrm{CH}_{3}\right)_{3}\right)\right]$ torna a reatividade com $\mathrm{H}_{2}$ mais complexa ${ }^{29}$. Estudos por IV in situ e determinação do teor de vanádio no sólido sugeriram que, à temperatura ambiente, ocorra a adição de $\mathrm{H}_{2}$ à ligação $\mathrm{V}=\mathrm{N}$ seguida por eliminação redutiva de neopentano (Esquema $6, i, i i)$. $\mathrm{O}$ aquecimento a $100{ }^{\circ} \mathrm{C}$ sob $\mathrm{H}_{2}$ provoca a hidrogenólise de ligações [V]-(OSi $\equiv)$ e liberação de espécies hidretos de vanádio (Esquema 6,iv). Simultaneamente, o hidrogênio seria adicionado ao complexo formando um intermediário [V]-H e t-butilamina. Esta, ao ser protonada por um hidrogênio ácido de um grupo silanol, formaria espécies iônicas de superfície (Esquema 6, iii) ${ }^{29}$.

\section{Termólise de complexos de superfície}

$\mathrm{O}$ aquecimento dos complexos $\left[(\equiv \mathrm{SiO})_{2} \mathrm{Cr}\left(\mathrm{CH}_{2} \mathrm{C}\left(\mathrm{CH}_{3}\right)_{3}\right)_{2}\right]$, $\left[(\equiv \mathrm{SiO})_{2} \mathrm{Cr}\left(\mathrm{CH}_{2} \mathrm{Si}\left(\mathrm{CH}_{3}\right)_{3}\right)_{2}\right]$ e $\left[(\equiv \mathrm{SiO})_{2} \mathrm{~V}\left(\mathrm{CH}_{2} \mathrm{Si}\left(\mathrm{CH}_{3}\right)_{3}\right)_{2}\right]$ conduz à formação dos respectivos alquilidenos de superfície, por abstração de hidrogênio $\alpha$, com liberação de neopentano ${ }^{41}$.

Os alquilidenos de superfície foram analisados por medidas de momento magnético, espectroscopia no IV in situ, quantificação dos gases liberados da reação de ancoramento, e reatividade química. Através de estudos cinéticos da termólise de cada complexo de superfície foi sugerido que a formação dos alquilidenos de cromo envolve duas etapas (Esquema 7). A primeira etapa seria a formação de um complexo Cr-hidreto, seguida por eliminação redutiva de alcano. $\mathrm{O}$ alquilideno de vanádio é formado em uma única etapa por abstração de hidrogênio $\alpha$ concertada ${ }^{41}$.

O complexo $\left[(\equiv \mathrm{SiO})_{2} \mathrm{Cr}\left(=\mathrm{CHC}\left(\mathrm{CH}_{3}\right)_{3}\right)\right]$ catalisa a homo e a copolimerização de etileno e 1-hexeno sem a adição de cocatalisadores ${ }^{41}$.

\begin{tabular}{|c|c|c|c|c|c|}
\hline Complexo precursor & $P_{\mathrm{H}_{2}}(\mathbf{a t m})$ & $\mathbf{T}\left({ }^{\circ} \mathbf{C}\right)$ & Hidreto & $v_{(\mathrm{MH})}\left(\mathbf{c m}^{-1}\right)$ & Ref. \\
\hline$\left[(\equiv \mathrm{SiO}) \mathrm{Zr}(\mathrm{Np})_{3}\right]$ & 0,5 & 150 & {$\left[(\equiv \mathrm{SiO})_{3} \mathrm{ZrH}\right]$} & 1635 & 4,20 \\
\hline $\begin{array}{l}{\left[(\equiv \mathrm{SiO}) \mathrm{Ta}\left(=\mathrm{CH}^{\mathrm{t}} \mathrm{Bu}\right)(\mathrm{Np})_{2}\right] \mathrm{e}} \\
{\left[(\equiv \mathrm{SiO})_{2} \mathrm{Ta}\left(=\mathrm{CH}^{\mathrm{t}} \mathrm{Bu}\right)(\mathrm{Np})\right]}\end{array}$ & 1,0 & 200 & {$\left[(\equiv \mathrm{SiO})_{2} \mathrm{TaH}\right]$} & 1830 & 38 \\
\hline$\left[(\equiv \mathrm{SiO}) \mathrm{Ti}(\mathrm{Np})_{3}\right]$ & 1,0 & 150 & {$\left[(\equiv \mathrm{SiO})_{3} \mathrm{TiH}\right]$} & $1706,1692,1679,1647$ & 40 \\
\hline
\end{tabular}

Tabela 1. Condições para a formação de hidretos de superfície e atribuição das bandas no infravermelho 


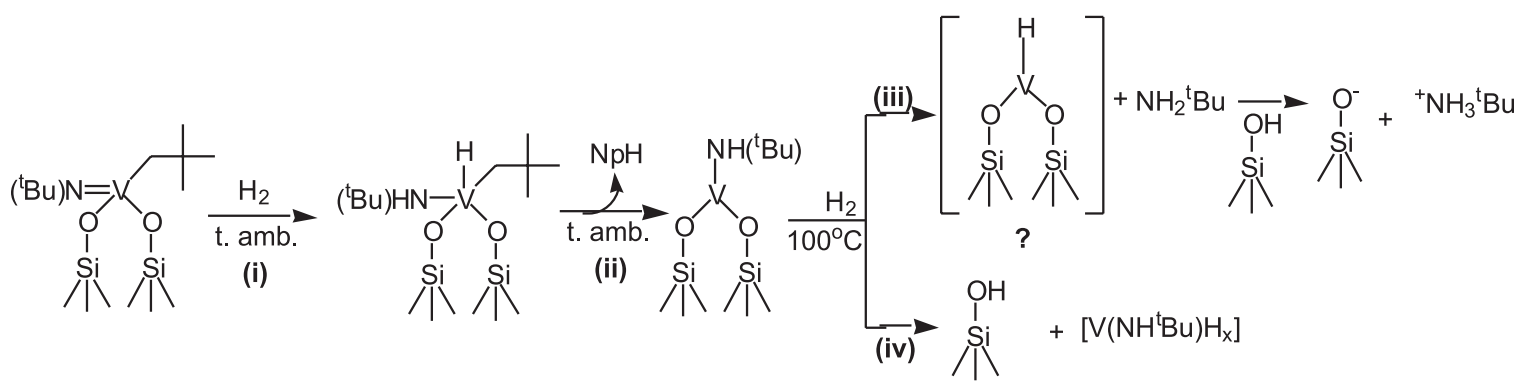

Esquema 6. Reatividade de $\left[(\equiv \mathrm{SiO})_{2} \mathrm{~V}\left(=\mathrm{N}^{t} \mathrm{Bu}\right)\left(\mathrm{CH}_{2} \mathrm{C}\left(\mathrm{CH}_{3}\right)_{3}\right)\right]$ com hidrogênio ${ }^{29}$
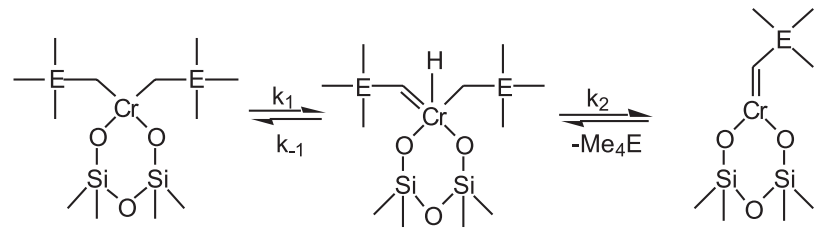

$\mathrm{E}=\mathrm{C}$ ou $\mathrm{Si}$

Esquema 7. Formação de $\left[(\equiv \mathrm{SiO})_{2} \mathrm{Cr}\left(=\mathrm{CHC}\left(\mathrm{CH}_{3}\right)_{3}\right)\right]^{41}$

\section{Formação de espécies discretas sobre a superfície}

Mediante reações controladas de oxidação e redução o complexo organometálico de superfície $\left[(\equiv \mathrm{SiO})_{2} \mathrm{Mo}\left(\mathrm{C}_{3} \mathrm{H}_{5}\right)_{2}\right]$ serve como precursor para a obtenção de espécies de molibdênio com sítios isolados, em diferentes estados de oxidação, importantes para uma variedade de reações catalíticas (Esquema 8$)^{15}$.

\section{CATÁLISE POR COMPLEXOS ORGANOMETÁLICOS DE SUPERFÍCIE}

Vários complexos organometálicos de superfície obtidos pela via Q.O.M.S. apresentam atividade catalítica. Destes, o catalisador derivado de $\left[\mathrm{Cr}(\mathrm{Cp})_{2}\right]$ suportado em sílica foi desenvolvido pela Union Carbide e é industrialmente aplicado para a produção de polietileno de alto peso molecular ${ }^{42}$.

A catálise por clusters de superfície já foi extensivamente revisada $^{1,9,15,34,35}$. Esses complexos apresentam atividade catalítica nas reações de deslocamento do gás d'água ("water gas shift -WGS") ${ }^{43}$, FischerTropsch $^{44}$, hidrogenação do etileno ${ }^{45}$ e de isomerização de olefinas ${ }^{46}$.

\section{Metátese catalítica de alcanos}

Uma nova reação catalítica foi observada com o hidreto de tântalo de superfície $\left[(\equiv \mathrm{SiO})_{2} \mathrm{TaH}\right]$, e foi chamada de metátese de alcanos em analogia à reação de metátese de olefinas ${ }^{39,47}$. Essa reação, sem precedentes na química organometálica clássica, ocorre pela clivagem e formação de ligações C-C de alcanos acíclicos, que são transformados numa mistura de homólogos de maior e menor cadeia de carbonos (Equação 12).

$\mathrm{C}_{\mathrm{n}} \mathrm{H}_{2 \mathrm{n}+2} \rightarrow \mathrm{C}_{\mathrm{n}+1} \mathrm{H}_{2(\mathrm{n}+\mathrm{i})+2}+\mathrm{C}_{\mathrm{n}-1} \mathrm{H}_{2(\mathrm{n}-\mathrm{i})+2} ; \mathrm{i}=1,2, \ldots$ (n-1)

O mecanismo ainda é desconhecido, mas existe a proposição de um ciclo catalítico baseado em observações experimentais (Esquema 9) ${ }^{39}$. De acordo com o monitoramento por IV e determinação dos produtos de reação, foi proposta a clivagem de uma ligação C-H do alcano, por metátese de ligações $\sigma$, produzindo hidrogênio e uma espécie [Ta]etila (2). Esta espécie ativaria a ligação $\mathrm{C}-\mathrm{C}$ do etano por metátese de ligações $\sigma$ (2'), liberando propano e formando uma espécie [Ta $]_{s}-\mathrm{CH}_{3}$ (3). Na última etapa, ocorreria a liberação de metano e a regeneração da espécie ativa (2) ${ }^{39}$.

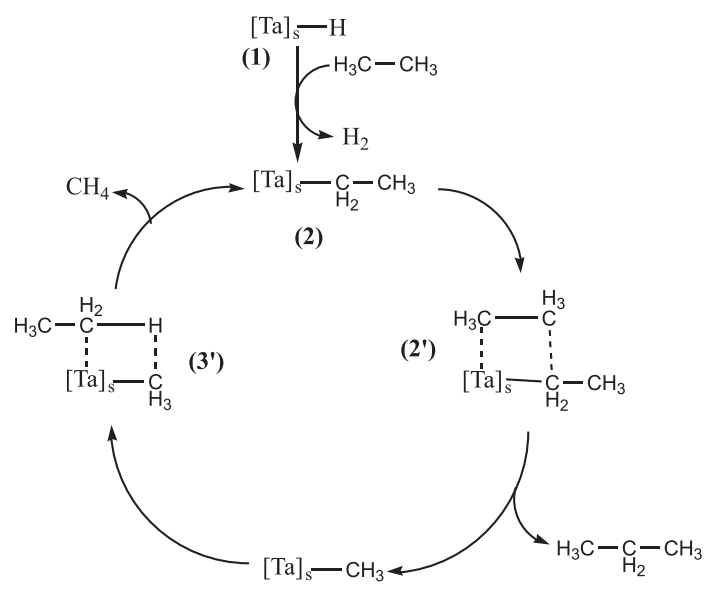

(3)

Esquema 9. Metátese do etano catalisada por $\left[(\equiv \mathrm{SiO})_{2} \mathrm{TaH}\right]^{39}$

\section{Hidrogenólise catalítica de alcanos}

A clivagem catalítica de ligações C-H e C-C de alcanos com




catalisadores convencionais requer altas temperaturas, tipicamente $500{ }^{\circ} \mathrm{C}{ }^{20}$. Entretanto, os complexos $\left[(\equiv \mathrm{SiO})_{3} \mathrm{MH}\right], \mathrm{M}=\mathrm{Zr}$, Hf e $\mathrm{Ti}$, efetuam a hidrogenólise de alcanos simples, como neopentano, isobutano e propano, a baixas temperaturas $\left(25\right.$ a $\left.150{ }^{\circ} \mathrm{C}\right)$ em presença de hidrogênio $0^{4,5,20,40,48}$.

$\mathrm{Na}$ hidrogenólise de neopentano com $\left[(\equiv \mathrm{SiO})_{3} \mathrm{MH}\right], \mathrm{M}=\mathrm{Zr}$ e Hf, inicialmente são formados isobutano e metano. A conversões mais elevadas, o isobutano é transformado em metano e propano que, por sua vez, é transformado em metano e etano. Como o etano não sofre a hidrogenólise, ao final obtém-se metano e etano numa relação 3:1. Traços de 2-metil-2-butano também são observados 5 .

O mecanismo proposto para a hidrogenólise do neopentano foi deduzido de reações estequiométricas efetuadas com $\left[(\equiv \mathrm{SiO})_{3} \mathrm{ZrH}\right]$ (Esquema 10) .



(3)

Esquema 10. Hidrogenólise de alcanos catalisada por $\left[(\equiv \mathrm{SiO})_{3} \mathrm{ZrH}\right]^{5}$

A primeira etapa da reação é a ativação da ligação $\mathrm{C}-\mathrm{H}$ do neopentano pelo hidreto de superfície, via metátese de ligações $\sigma$ $(\mathbf{2} \rightarrow \mathbf{3})$. A posterior transferência de metila $\beta$ produz isobuteno e $[\mathrm{Zr}]_{\mathrm{s}}$ $\mathrm{Me}(\mathbf{3} \rightarrow \mathbf{4})$. O isobuteno sofre hidrogenação pelo hidreto de superfície, produzindo isobutano $(\mathbf{2} \rightarrow \mathbf{1} \rightarrow \mathbf{2})$. A última etapa do ciclo $(\mathbf{4} \rightarrow \mathbf{2})$ é a hidrogenólise do fragmento de superfície $[\mathrm{Zr}]_{\mathrm{s}}-\mathrm{Me}$, produzindo metano. O 2-metilbutano é formado por adição do tipo Markovnikov do isobuteno ao fragmento $[\mathrm{Zr}]_{\mathrm{s}}-\mathrm{Me}$, seguida pela hidrogenólise da ligação $[\mathrm{Zr}]_{\mathrm{s}}$-alquila (ciclo interno) . $^{5}$

$\mathrm{A}$ ativação catalítica de ligações $\mathrm{CH}$ de alcanos à baixa temperatura nunca foi observada com hidretos de zircônio moleculares, provavelmente porque, em geral, são complexos de 16 elétrons, estabilizados por dois ligantes ciclopentadienila ${ }^{49}$. $\mathrm{O}$ hidreto de superfície sendo, formalmente, uma espécie de 8 elétrons, coordenativamente insaturada, com o centro de zircônio mais eletrofílico do que em análogos moleculares, possibilita a reação com o alcano. Também deve ser considerada a coordenação do zircônio à superfície, que estabilizaria o complexo evitando a decomposição bimolecular, provável em espécies moleculares ${ }^{5}$.

Diferentemente dos complexos $\left[(\equiv \mathrm{SiO})_{3} \mathrm{ZrH}\right]$ e $\left[(\equiv \mathrm{SiO})_{3} \mathrm{HfH}\right]$ que produzem, a partir de neopentano, metano e etano numa razão de 3:1, o complexo $\left[(\equiv \mathrm{SiO})_{3} \mathrm{TiH}\right]$ os produz numa razão de $1: 1$. Este resultado foi atribuído à isomerização do alcano durante o ciclo de hidrogenólise e ocorreria quando a espécie metal-alquila estivesse formada, podendo sofrer a eliminação de uma metila na posição $\beta$. Nesse caso, o intermediário metal-alquila persistiria por um tempo suficiente para que ocorresse a rotação da olefina e sua reinserção à ligação metal-carbono (Equação 13) ${ }^{40}$.

$$
\mathrm{Ti}-\mathrm{H} \stackrel{+\mathrm{NpH}}{-\mathrm{H}_{2}} \rightarrow \mathrm{Ti} \longrightarrow \stackrel{\mathrm{Ti}}{\rightarrow} \stackrel{\mathrm{CH}_{3}}{\stackrel{+2 \mathrm{H}_{2}}{\longrightarrow}}=\mathrm{M}-\mathrm{H}+\mathrm{CH}_{4}+\mathrm{CH}\left(\mathrm{CH}_{3}\right)_{3}
$$

\section{Polimerização de olefinas e degradação de polímeros}

O hidreto de zircônio $\left[(\equiv \mathrm{SiO})_{3} \mathrm{ZrH}\right]$ na ausência de hidrogênio polimeriza olefinas como etileno e propileno. Interessantemente, o mesmo complexo é capaz de transformar polietileno em metano e etano, sob hidrogênio a $150{ }^{\circ} \mathrm{C}{ }^{50}$.

A polimerização do etilleno e propileno foi verificada por espectroscopia no IV in situ. Quando o complexo $\left[(\equiv \mathrm{SiO})_{3} \mathrm{ZrH}\right]$ foi exposto a etileno (200 Torr, temperatura ambiente) a banda relativa ao $v_{(\mathrm{zr}-\mathrm{H})}$ desapareceu com o aparecimento de bandas características de polietileno ${ }^{50}$. O mecanismo consiste na inserção da olefina na ligação $[\mathrm{Zr}]_{\mathrm{s}}-\mathrm{H}$ e posteriores inserções em ligações $[\mathrm{Zr}]_{\mathrm{s}}$-alquila ${ }^{36}$.

Considerando que as poliolefinas são alcanos de cadeia longa, Dufaud e Basset mostraram que o hidreto de zircônio suportado em sílica-alumina, em presença de hidrogênio, é capaz de clivar ligações $\mathrm{C}$-C de polietileno ou polipropileno, produzindo oligômeros saturados de cadeias curtas $\mathrm{C}_{10}-\mathrm{C}_{17}$ (diesel) ou, eventualmente, alcanos mais leves, $<\mathrm{C}_{9}$, etano e metano ${ }^{50}$.

Amostras comerciais de polímeros foram degradadas com o catalisador em condições brandas $\left(150{ }^{\circ} \mathrm{C}\right.$ e 1 atm de $\left.\mathrm{H}_{2}\right) . \mathrm{Em} 5 \mathrm{~h}$ de reação, o catalisador converte polietileno de baixo peso molecular $\left(\mathrm{C}_{18}\right.$ a $\left.\mathrm{C}_{50}\right)$ a alcanos na faixa de $\mathrm{C}_{1}$ a $\mathrm{C}_{9}(84 \%)$ e na faixa de $\mathrm{C}_{10} \mathrm{a} \mathrm{C}_{18}$ (16\%). Após $62 \mathrm{~h}$ de reação, foram obtidos somente alcanos leves $(<$ $\mathrm{C}_{6}$ ). Com polietileno de baixa densidade e de alta massa molecular $(\mathrm{M}=125000)$, após $10 \mathrm{~h}$ de reação, atingiu-se $100 \%$ de conversão a alcanos na faixa de $\mathrm{C}_{1}$ a $\mathrm{C}_{9}$. Polipropileno isotático $(\mathrm{M}=250000)$ foi clivado a $190{ }^{\circ} \mathrm{C}$, na ausência de solvente. Após 15 h de reação, $40 \%$ do polipropileno foi convertido em alcanos leves $\left(<\mathrm{C}_{7}\right)^{36}$.

$\mathrm{O}$ mecanismo proposto foi baseado em etapas elementares conhecidas na química de organometálicos (Esquema 11) ${ }^{36}$.

A primeira etapa é a ativação não seletiva de uma ligação $\mathrm{CH}$ da cadeia polimérica por metátese de ligação $\sigma$ com liberação de hidrogênio $(\mathbf{1} \rightarrow \mathbf{2})$. A segunda etapa é a transferência de uma alquila $\beta$ de parte da cadeia polimérica $(\mathbf{2} \rightarrow \mathbf{3})$, seguida de eliminação do polímero clivado, gerando um hidreto de zircônio $(\mathbf{3} \rightarrow \mathbf{4})$. A etapa $(\mathbf{2} \rightarrow \mathbf{3})$ pode

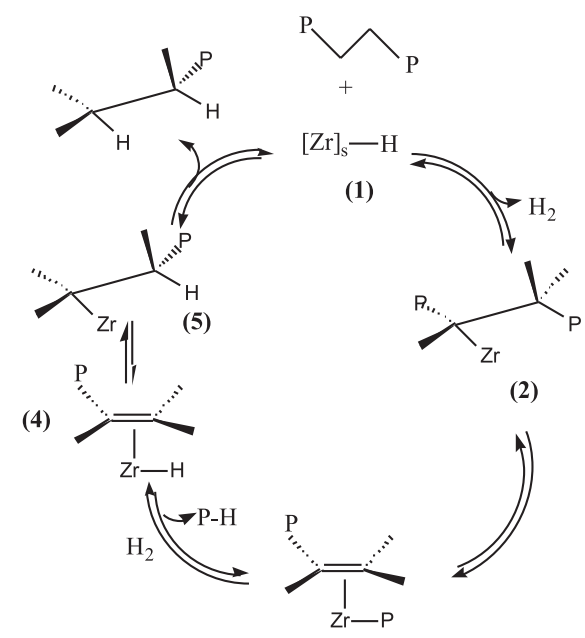

(3)

Esquema 11. Mecanismo para a degradação de polietileno. $P=$ cadeia polimérica $^{36}$ 
ser considerada como o reverso da etapa chave de inserção de uma olefina a uma ligação metal-alquila, na polimerização Ziegler-Natta. Depois ocorre a hidrogenação da ligação dupla e restauração do hidreto de superfície $(\mathbf{5} \rightarrow \mathbf{1})^{36}$.

\section{Metátese de olefinas}

Basicamente duas estratégias têm sido utilizadas para sintetizar catalisadores para a metátese de olefinas via Q.O.M.S. A primeira consiste em utilizar complexos organometálicos de metais de transição que, tipicamente, apresentam boa atividade em catálise heterogênea como molibdênio ${ }^{51}$, tungstênio ${ }^{52}$ e rênio ${ }^{53}$. Por exemplo, o complexo $\left[\left(\mathrm{CH}_{3}\right) \mathrm{ReO}_{3}\right]$ é inativo para a metátese de olefinas acíclicas, mas, quando suportado em nióbia, apresenta alta atividade. Pelo menos três diferentes espécies rênio-metila são formadas sobre a superfície. O catalisador pode ser reciclado, atingindo a conversão de, no mínimo, 2500 equivalentes de $c i s-2$-penteno ${ }^{53}$. A atividade foi correlacionada com a acidez de Lewis do suporte ${ }^{53}$. A formação de 2,5-dimetil-2-hexeno, isobuteno, 3-metil-1-buteno e, provavelmente, 5-metil-2-hexeno, como produtos de reação de $\left[\left(\mathrm{CH}_{3}\right) \mathrm{ReO}_{3}\right] / \mathrm{Nb}_{2} \mathrm{O}_{5}$ com trans-2,5-dimetil-3-hexeno, sugerem que o primeiro metalcarbeno é formado através de um mecanismo $\pi$-alila (Esquema12) ${ }^{53}$.

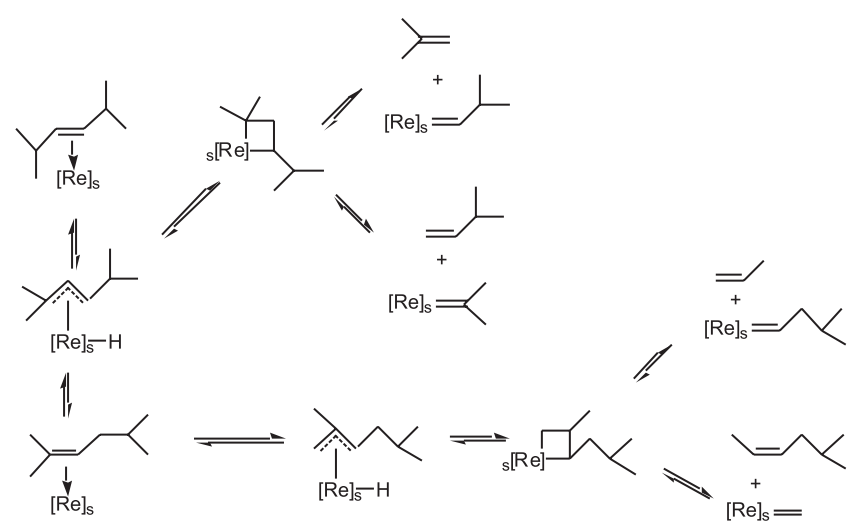

Esquema 12. Mecanismo $\pi$-alila de formação do metal-carbeno $\mathrm{em}\left[\left(\mathrm{CH}_{3}\right) \mathrm{ReO}_{3}\right] / \mathrm{Nb}_{2} \mathrm{O}_{5}{ }^{53}$

A segunda estratégia consiste em sintetizar, diretamente sobre a superfície, complexos metal-alquilideno utilizando como modelo reações da química molecular. Esta estratégia está embasada no conhecimento de que a espécie cataliticamente ativa seja um complexo metal-carbeno e que vários complexos $\mathrm{M}$-alquilideno iniciam a reação em fase homogênea ${ }^{54}$.

Assim, foram sintetizados complexos M-alquilidenos de superfície relativamente bem definidos, como $\left[(\equiv \mathrm{SiO})_{2} \mathrm{~W}\left(=\mathrm{CH}{ }^{\mathrm{B} u}\right) \mathrm{X}\right]^{25}, \mathrm{X}=$ $\mathrm{CH}_{2}{ }^{ } \mathrm{Bu}$ ou $\mathrm{Cl},\left[(\equiv \mathrm{SiO}) \mathrm{Mo}\left(=\mathrm{CH}{ }^{\mathrm{B}} \mathrm{Bu}\right)(=\mathrm{NH})\left(\mathrm{CH}_{2}{ }^{\mathrm{t}} \mathrm{Bu}\right)\right]^{27}$, ativos em reações de metátese de olefinas, em que seus precursores moleculares não apresentam atividade. Recentemente foi preparado o complexo bem definido de rênio suportado em sílica, $\left[(\equiv \mathrm{SiO}) \mathrm{Re}\left(\equiv \mathrm{C}^{t} \mathrm{Bu}\right)\right.$ $\left.\left(=\mathrm{CH}{ }^{\mathrm{t}} \mathrm{Bu}\right)\left(\mathrm{CH}_{2}{ }^{ } \mathrm{Bu}\right)\right]$, ativo em metátese de olefinas e de alquinos ${ }^{55}$, sendo sua atividade comparável à dos melhores catalisadores à base de rênio ${ }^{56}$

Os complexos $\left[\mathrm{W}\left(\equiv \mathrm{C}{ }^{\mathrm{t}} \mathrm{Bu}\right)(\mathrm{X})_{3}\right], \mathrm{X}=\mathrm{CH}_{2}{ }^{\mathrm{t}} \mathrm{Bu}$ ou $\mathrm{Cl}(\mathrm{dme})$, dme =dimetoxietano, e $\left[\mathrm{WO}(\mathrm{ONp}) \mathrm{Np}_{3}\right]$ suportados em $\mathrm{Nb}_{2} \mathrm{O}_{5}$ (Esquema 4) formam catalisadores altamente ativos para a metátese de cis-2penteno ${ }^{26,30,57}$. De acordo com estudos de reatividade química, a atividade foi atribuída às espécies [W]-neopentilideno de superfície e espécies reduzidas de W. As últimas gerariam o primeiro metal-carbeno via me- canismo $\pi$-alila ${ }^{26,30,57}$. Diferentemente desses complexos, que tornam-se ativos ao serem suportados em $\mathrm{Nb}_{2} \mathrm{O}_{5}$, os complexos $\left[\mathrm{Mo}\left(=\mathrm{CHCPhMe}_{2}\right)\right.$ $\left.(=\mathrm{NAr})\left(\mathrm{OC}(\mathrm{Me})_{3}\right)_{2}\right]$ e $\left[\mathrm{Mo}\left(=\mathrm{CHCPhMe}_{2}\right)(=\mathrm{NAr})\left(\mathrm{OC}(\mathrm{Me})\left(\mathrm{CF}_{3}\right)_{2}\right)_{2}\right]$, quando suportados em sílica ou sílica-alumina têm suas atividades diminuídas na metátese do cis-2-penteno. Este resultado foi atribuído à interação ácido-base de Lewis do complexo com os grupos silanóis, que diminui a eletrofilicidade do metal ${ }^{32}$. Na polimerização metatética por abertura de anel (ROMP, "Ring Closing Metathesis Polymerization") do norborneno foram obtidos polímeros com polidispersidades maiores do que 1 e estereosseletividades diferentes dos catalisadores homogêneos, supostamente devido ao impedimento estérico causado pela superfície e/ou à perda de sítios desocupados na esfera de coordenação dos complexos ancorados ${ }^{32}$.

\section{Epoxidação assimétrica de álcoois alílicos}

Este é um exemplo que demonstra que fatores estéricos e eletrônicos devem ser considerados quando se pretende sintetizar catalisadores por Q.O.M.S.

Em fase homogênea, alcóxidos de titânio, em presença de um indutor de quiralidade e de um oxidante (catalisadores de Sharpless) catalisam a epoxidação assimétrica de álcoois alílicos ${ }^{58}$. No mecanismo aceito para a reação, os quatro elétrons d do Ti estão envolvidos em ligações $\sigma$ : duas ligações $\sigma$ com os átomos de oxigênio do grupo quelato tartarato, uma ligação com o grupo alcoxi-alil e uma ligação $\sigma / \pi$ com o grupo t-butilperoxo ${ }^{59}$. Essa esfera de coordenação descarta a síntese de catalisadores sólidos à base de alcóxidos de titânio, pois se o $\mathrm{Ti}^{\mathrm{IV}}$ estivesse coordenado à superfície via ligação Si-O-Ti, não seria possível acomodar todos os ligantes. Efetivamente, catalisadores de $\left[\mathrm{Ti}\left(\mathrm{O}^{\mathrm{i} P r}\right)_{4}\right]$ suportados em sílica apresentaram baixa atividade e, praticamente, nenhuma enantiosseletividade na epoxidação de 2-propen-1-ol e trans-2-hexen-1-ol ${ }^{60}$.

Por outro lado, o tântalo, metal do grupo 5 , coordenado à superfície poderia acomodar os ligantes. Assim, foram sintetizados etóxidos de tântalo por reação de complexos organometálicos de superfície com etanol (Equação 14) ${ }^{60}$.

$$
\begin{aligned}
& {\left[(\equiv \mathrm{SiO}) \mathrm{Ta}\left(=\mathrm{CH}^{\mathrm{t}} \mathrm{Bu}\right)\left(\mathrm{CH}_{2}{ }^{\mathrm{t}} \mathrm{Bu}\right)_{2}\right]+\mathrm{EtOH}+\left[(\equiv \mathrm{SiO}) \mathrm{Ta}(\mathrm{OEt})_{4}\right]} \\
& {\left[(\equiv \mathrm{SiO})_{2} \mathrm{Ta}\left(={ }_{\mathrm{CH}}^{+} \mathrm{Bu}\right)\left(\mathrm{CH}_{2}{ }^{\mathrm{t}} \mathrm{Bu}\right)\right] \quad \stackrel{+\left(\mathrm{CH}_{3}{ }^{\mathrm{t}} \mathrm{Bu}\right)}{\longrightarrow} \stackrel{+}{\left.\left.{ }_{[(\equiv \mathrm{SiO})}\right)_{2} \mathrm{Ta}(\mathrm{OEt})_{3}\right]}}
\end{aligned}
$$

Esses sólidos apresentaram boa atividade e enantiosseletividade para a epoxidação catalítica de 2-propen-1-ol e trans-2-hexen-1-ol. O análogo molecular $\left[\mathrm{Ta}(\mathrm{OEt})_{5}\right]$ não apresenta atividade devido a uma possível dimerização do complexo no meio reacional ${ }^{60}$. A dimerização no catalisador sólido não ocorre pois são formadas espécies monoméricas de Ta bem dispersas na superfície, que são cataliticamente ativas. Por exemplo, na epoxidação do 2-propen-1-ol, usando como indutor quiral o (+)-diisopropil-tartarato e como oxidante t-butilhidroperóxido, foi produzido (s)-glicidol com número de rotação (NR) de 28 e $84 \%$ de excesso enantiomérico (e.e.). Nas mesmas condições, o catalisador homogêneo $\left[\mathrm{Ti}(\mathrm{O}-i \mathrm{Pr})_{4}\right]$ produz (s)-glicidol com $\mathrm{NR}=$ 15 e $80 \%$ e.e. $\mathrm{Na}$ epoxidação do trans-2-hexen-1-ol, embora o catalisador sólido seja um pouco menos efetivo $(\mathrm{NR}=10,90 \%$ e.e.) do que o homogêneo ( $\mathrm{NR}=16,96 \%$ e.e.), é possível reciclá-lo sem grandes perdas de atividade $(\mathrm{NR}=8,93 \% \text { e.e. })^{60}$.

\section{CONCLUSÃO E PERSPECTIVAS}

O interesse principal da Q.O.M.S. é a racionalização da química de complexos organometálicos em relação à superfície de óxidos inorgânicos, zeólitas e metais. Neste sentido, já foi mostrado que a 
síntese de complexos organometálicos de superfície segue as regras da química em solução. O maior desafio da Q.O.M.S., no âmbito da catálise, é a síntese de sistemas catalíticos heterogenizados que, além de serem bem definidos e caracterizados, sejam também eficientes e estáveis para uma determinada reação catalítica. Desta maneira, não se obteria apenas um bom sistema catalítico, mas, também, seria possível contribuir para a elucidação das espécies ativas e dos mecanismos de reação de catalisadores heterogêneos convencionais. Entretanto, a maioria dos estudos ainda se concentra na preparação de sistemas bem definidos, enquanto que a estabilidade e a eficiência dos catalisadores assim obtidos ainda deixam muito a desejar. Com a utilização das reações de superfície já conhecidas, mostradas aqui, ou explorando novas reações que tenham precedentes na química em solução, novos complexos de superfície poderão ser sintetizados e aplicados a um maior número de reações catalíticas. A tendência desta química parece estar direcionada ao uso de novos complexos organometálicos e à exploração de outros suportes, como sólidos mesoporosos, dendrímeros, fulerenos, etc.

\section{AGRADECIMENTOS}

À FAPESP pelo apoio financeiro e pela bolsa de doutorado concedida (S. I. Wolke).

\section{REFERÊNCIAS}

1. Basset, J.-M.; Gates, B. C.; Candy, J. P.; Choplin, A.; Leconte, M.; Quignard, F.; Santini, C.; Surface Organometallic Chemistry: Molecular Approaches to Surface Catalysis, Kluwer Academic Publishers: Amsterdam, 1988.

2. Yermakov, Y. I.; Pure Appl. Chem. 1980, 52, 2075.

3. Candlin, J. P.; Thomas, H.; Adv. Chem. Ser. 1974, 132, 212.

4. Lécuyer, C.; Quignard, F.; Choplin, A.; Olivier, D.; Basset, J.-M.; Angew. Chem., Int. Ed. 1991, 30, 1660.

5. Quignard, F.; Lécuyer, C.; Choplin, A.; Olivier, D.; Basset, J.-M.; J. Mol. Catal. 1992, 74, 353

6. Yermakov, Y. I.; Kuznetzov, B. N.; Zakharov, V. A.; Catalysis by Supported Metal Complexes, Elsevier: Amsterdam, 1981.

7. Basset, J.-M.; Lefebvre, F.; Santini, C.; Coord. Chem. Rev. 1998, 180, 1703.

8. Lefort, L.; Chabanas, M.; Maury, O.; Meunier, D.; Copéret, C.; ThivolleCazat, J.; Basset, J.-M.; J. Organomet. Chem. 2000, 593-594, 96.

9. Basset, J.-M.; Choplin, A.; J. Mol. Catal. 1983, 21, 95; Bailey, D. C.; Langer, S. H.; Chem. Rev. 1981, 81, 109; Lamb, H. H.; Gates, B. C.; Knözinger, H.; Angew. Chem., Int. Ed. 1988, 27, 1127; Zecchina, A.; Areán, C. O.; Catal. Rev.-Sci. Eng. 1993, 35, 261.

10. Correa, F.; Nakamura, R.; Stimson, R. E.; Burwell Jr., R. L.; Shriver, D. F.; J. Am. Chem. Soc. 1980, 102, 5112.

11. Lamb, H. H.; Gates, B. C.; J. Am. Chem. Soc. 1986, 108, 81.

12. Hugues, F.; Basset, J.-M.; Ben Taarit, Y.; Choplin, A.; Primet, M.; Rojas, D.; Smith, A. K.; J. Am. Chem. Soc. 1982, 104, 7020; Psaro, R.; Dossi, C.; Ugo, R.; J. Mol. Catal. 1983, 21, 331; Pierantozzi, R.; Valagene, E. G.; Nordquist, A. F.; Dyer, P. N.; J. Mol. Catal. 1983, 21, 189; Kuznetsov, V. L.; Bell, A. T.; Yermakov, Y. I.; J. Catal. 1980, 65, 374.

13. Besson, B.; Moraweck, B.; Smith, A. K.; Basset, J.-M.; Psaro, R.; Fusi, A.; Ugo, R.; J. Chem. Soc., Chem. Commun. 1980, 569; Psaro, R.; Ugo, R.; Besson, B.; Zanderighi, G. M.; Smith, A. K.; Basset, J.-M.; J. Organomet. Chem. 1981, 213, 215.

14. Deeba, M.; Gates, B. C.; J. Catal. 1981, 67, 303; Cook, S. L.;. Evans, J; McNulty, G. S.; Greaves, G. N.; J. Chem. Soc., Dalton Trans. 1986, 7; Duivenvoorden, F. B. M.; Koningsberger, D. C.; Uh, Y. S.; Gates, B. C.; J. Am. Chem. Soc. 1986, 108, 6254; Puga, J.; Fehlner, T. P.; Gates, B. C.; Braga, D.; Grepioni, F.; Inorg. Chem. 1990, 29, 2376; Deeba, M.; Streusand, B. J.; Schrader, G. L.; Gates, B. C.; J. Catal. 1981, 69, 218.

15. Ballard, D. G. H.; Adv. Catal. 1973, 23, 263; Yermakov, Y. I.; Catal. Rev.Sci. Eng. 1976, 13, 77; Iwasawa, Y.; Adv. Catal. 1987, 35, 187.

16. Dufour, P.; Houtman, C.; Santini, C. C.; Nédez, C.; Basset, J.-M.; Hsu, L. Y.; Shore, S. G.; J. Am. Chem. Soc. 1992, 114, 4248; Ward, M. D.; Harris, T. V.; Schwartz, J.; J. Chem. Soc., Chem. Commun. 1980, 357; Iwasawa, Y.; Sato, H.; Chem. Lett. 1985, 507; Fischer, H. E.; King, S. A.; Bronnimann, C. E.; Schwartz, J.; Langmuir 1993, 9, 391; Ward, M.D.; Schwartz, J.; J. Am. Chem. Soc. 1981, 103, 5253; Foley, H.C.; DeCanio,
S.J.; Tau, K.D.; Chao, K.J.; Onuferko, J.H.; Dybowski, C.; Gates, B. C.; J. Am. Chem. Soc. 1983, 105, 3074.

17. Mckenna ,W. P.; Eyring, E. M.; J. Mol. Catal. 1985, 29, 363; Griffe, B.; Sierraalta, A.; Ruette, F.; Brito, J. L.; J. Mol. Catal. A: Chem. 2001, 168, 265.

18. Aigler, J. M.; Brito, J. L.; Leach, P. A.; Houalla, M.; Proctor, A.; Cooper, N. J.; Hall, W. K.; Hercules, D. M.; J. Phys. Chem. 1993, 97, 5699.

19. Quignard, F.; Lécuyer, C.; Bougault, C.; Lefebvre, F.; Choplin, A.; Olivier, D.; Basset, J.-M.; Inorg. Chem. 1992, 31, 928.

20. Corker, J.; Lefebvre, F.; Lécuyer, C.; Dufaud, V.; Quignard, F.; Choplin, A.; Evans, J.; Basset, J.-M.; Science 1996, 271, 966.

21. Jezequel, M.; Dufaud, V.; Ruiz-Garcia, M. J.; Carrillo-Hermosilla, F.; Neugebauer, U.; Niccolai, G. P; Lefebvre, F.; Bayard, F.; Corker, J.; Fiddy, S.; Evans, J.; Broyer, J.-P.; Malinge, J.; Basset, J.-M.; J. Am. Chem. Soc. 2001, 123, 3520

22. Dufaud, V.; Niccolai, G. P; Thivolle-Cazat, J.; Basset, J.-M.; J. Am. Chem. Soc. 1995, 117, 4288.

23. Schrock, R. R.; J. Am. Chem. Soc. 1976, 98, 5399.

24. Freudenberger, J. H.; Schrock, R. R.; Organometallics 1985, 4, 1937.

25. Weiss, K; Lössel, G.; Angew. Chem., Int. Ed. 1989, 28, 62.

26. Buffon, R.; Leconte, M.; Choplin, A.; Basset, J.-M.; J. Chem. Soc., Dalton Trans. 1994, 1723.

27. Herrmann, W. A.; Stumpf, A. W.; Priermeier, T.; Bogdanovic, S.; Dufaud, V.; Basset, J.-M.; Angew. Chem., Int. Ed. 1996, 35, 2803.

28. Wolke, S. I.; Tese de Doutorado, Universidade Estadual de Campinas, Brasil, 2000.

29. Wolke, S. I.; Buffon, R.; Rodrigues Filho, U. P.; J. Organomet. Chem. 2001, $625,101$.

30. Buffon, R.; Leconte, M.; Choplin, A.; Basset, J.-M; Anais do $7^{\circ}$ Seminário Brasileiro de Catálise, Instituto Brasileiro do Petróleo, Rio de Janeiro, Brasil, 1993.

31. Kress, J. R. M.; Russel, M. J. M.; Wesolek, M. G.; Osborn, J. A.; J. Chem. Soc., Chem. Commun. 1980, 431; Kress, J. R. M.; Wesolek, M. G.; Le Ny, J. M.; Osborn, J. A.; J. Chem. Soc., Chem. Commun. 1981, 1039.

32. Wolke, S. I.; Buffon, R.; J. Mol. Catal. A: Chem. 2000, 160, 181.

33. Fox, H. H.; Lee, J. K.; Park, L. Y.; Schrock, R. R.; Organometallics 1993, 12,759 .

34. Scott, S. L.; Basset, J.-M.; J. Mol. Catal. 1994, 86, 5

35. Basset, J.-M.; Candy, J. P.; Choplin, A.; Didillon, B.; Quignard, F.; Théolier, A. Em Perspectives in Catalysis; Thomas, J. M.; Zamaraev, K., eds.; Blackwell: Oxford, 1991, p.125; Scott, S. L.; Basset, J.-M.; Niccolai, G. P.; Santini, C. C.; Candy, J. P.; Lécuyer, C.; Quignard, F.; Choplin; A.; New J. Chem. 1994, 18, 115; Lefebvre, F.; Candy, J. P.; Santini, C. C.; Basset, J.-M.; Top. Catal. 1997, 4, 211; Choplin, A.; Quignard, F.; Coord. Chem. Rev. 1998, 180, 1679.

36. Lefebvre, F.; Thivolle-Cazat, J.; Dufaud, V.; Niccolai, G. P; Basset, J.-M.; Appl. Catal., A 1999, 182, 1.

37. Quignard, F.; Lécuyer, C.; Choplin, A.; Basset, J.-M.; J. Chem. Soc., Dalton Trans. 1994, 1153; Quignard, F.; Choplin, A.; Basset, J.-M.; J. Chem. Soc., Dalton Trans. 1994, 2411.

38. Vidal, V.; Théolier, A.; Thivolle-Cazat, J.; Basset, J.-M.; Corker, J.; J. Am. Chem. Soc. 1996, 118, 4595

39. Vidal, V.; Théolier, A.; Thivolle-Cazat, J.; Basset, J.-M.; Science 1997, 276, 99.

40. Rosier, C.; Niccolai, G. P.; Basset, J.-M.; J. Am. Chem. Soc. 1997, 119, 12408.

41. Ajjou, J.A.N.; Scott, S.L.; Paquet, V.; J. Am. Chem. Soc. 1998, 120, 415; Ajjou, J.A.N.; Rice, G. L.; Scott, S. L.; J. Am. Chem. Soc. 1998, 120, 13436; Ajjou, J.A.N.; Scott, S.L.; J. Am. Chem. Soc. 2000, 122, 8968.

42. Karol, F. J.; Karapinka, G. L.; Johnson, R. N.; Wu, C.; Carrick, W. L.; Dow, A.W.; J. Polym. Sci., Part A: Polym. Chem. 1972, 10, 2621.

43. Basset, J.-M.; Théolier, A.; Commereuc, D.; Chauvin, Y.; J. Organomet. Chem. 1985, 279, 147.

44. Ichikawa, M.; J. Chem. Soc., Chem. Commun. 1978, 566; Smith, A. K.; Théolier, A.; Basset, J.-M.; Ugo, R.; Commereuc, D.; Chauvin, Y.; J. Am. Chem. Soc. 1978, 100, 2590; Vanhove, D.; Makambo, P.; Blanchard, M.; J. Chem. Soc., Chem. Commun. 1979, 605.

45. Besson, B.; Choplin, A.; D’Ornelas, L.; Basset, J.-M.; J. Chem. Soc., Chem. Commun. 1982, 843; Choplin, A.; Besson, B.; D’Ornelas, L.; SanchezDelgado, R.; Basset, J.-M.; J. Am. Chem. Soc. 1988, 110, 2783.

46. Krause, T.R.; Davies, M. E.; Lieto, J.; Gates, B. C.; J. Catal. 1985, 94, 195; Scott, J. P.; Budge, J. R.; Rheingold, A. L.; Gates, B. C.; J. Am. Chem. Soc. 1987, 109, 7736.

47. Maury, O.; Lefort, L.; Vidal, V.; Thivolle-Cazat, J.; Basset, J.-M.; Angew. Chem., Int. Ed. 1999, 38, 1952.

48. Ornelas, L.; Reyes, S.; Quignard, F.; Choplin, A.; Basset, J.-M.; Chem. Lett. 1993, 1931. 
49. Manriquez, J. M.; McAlister, D. R.; Sanner, R. D.; Bercaw, J. E.; J. Am. Chem. Soc. 1976, 98, 6733.

50. Dufaud, V., Basset, J.-M.; Angew. Chem., Int. Ed. 1998, 37, 806.

51. Iwasawa, Y.; Ichinose, H.; Ogasawara, S.; Soma, M.; J. Chem. Soc., Faraday Trans. 1 1981, 77, 1763; Iwasawa, Y.; Kubo, H.; Hamamura, H.; J. Mol. Catal. 1985, 28, 191.

52. Kuznetsov, B. N.; Startsev, A. N.; Yermakov, Y. I.; J. Mol. Catal. 1980, 8, 135.

53. Buffon, R.; Auroux, A.; Lefebvre, F.; Leconte, M.; Choplin, A.; Basset, J.-M.; Herrmann, W. A.; J. Mol. Catal. 1992, 76, 287.; Buffon, R.; Choplin, A.; Leconte, M.; Basset, J.-M.; Touroude, R.; Herrmann, W. A.; J. Mol. Catal. 1992, 72, L7.
54. Hérisson, J. L.; Chauvin, Y.; Makromol. Chem. 1970, 141, 161

55. Chabanas, M.; Baudouin, A.; Copéret, C.; Basset, J.-M.; J. Am. Chem. Soc. 2001, 123, 2062.

56. Ivin, K. J.; Mol, J. C.; Olefin Metathesis and Metathesis Polimeryzation, Academic Press: London, 1997.

57. Buffon, R.; Leconte, M.; Choplin, A.; Basset, J.-M.; J. Chem. Soc., Chem. Commun. 1993, 361.

58. Katsuki, T.; Sharpless, K. B.; J. Am. Chem. Soc. 1980, 102, 5974.

59. Finn, M. G.; Sharpless, K. B.; J. Am. Chem. Soc. 1991, 113, 113.

60. Meunier, D.; Piechaczyk, A.; Mallmann, A.; Basset, J.-M.; Angew. Chem. Int. Ed. 1999, 38, 3540. 